\title{
Ray Trace Study for Visible Spectroscopy Reference System (VSRS) Diagnostics in ITER
}

\author{
Shin KAJITA, Maarten DE BOCK ${ }^{1)}$, Michel DESJARDINS ${ }^{2)}$ and Robin BARNSLEY ${ }^{1)}$ \\ Institute of Materials and Systems for Sustainability, Nagoya University, Nagoya 464-8603, Japan \\ 1) ITER Organization, Route de Vinon-sur-Verdon, CS 90 046, 13067 St. Paul Lez Durance Cedex, France \\ ${ }^{2)}$ Topequa, Avenue Campagne Barielle, 13013 Marseille, France
}

(Received 17 December 2018 / Accepted 24 January 2019)

\begin{abstract}
The visible spectroscopy reference system (VSRS) diagnostic will be used to measure the continuum visible light emitted from the plasma in ITER. Because the field of view of the VSRS system is aligned to a hole on a blanket module with a retroreflector on the bottom, which will be shared with an interferometer measurement, it has a potential to be free from the stray light, which may disturb signals for optical diagnostics in ITER. In this study, performance of the stray light reduction in the VSRS is investigated using a ray tracing simulation. We investigated the influences of the reflection property of the retroreflector and first mirror for the signal and stray light intensity.
\end{abstract}

(C) 2019 The Japan Society of Plasma Science and Nuclear Fusion Research

Keywords: ITER, visible spectroscopy reference system (VSRS), spectroscopy, stray light, ray tracing

DOI: $10.1585 /$ pfr. 14.1405042

\section{Introduction}

The primary role of the visible spectroscopy reference system (VSRS) diagnostic is to measure the continuum visible light emitted by the ITER plasma, which provides information on the impurity content of the plasma and the potential transparency for neutral beam heating power (hence providing a warning for potential heating beam shine through that could damage the ITER walls). This measurement of the continuum visible light is a basic control requirement, without which the ITER device is not allowed to operate. As a result, the VSRS design shall focus on reliability and robustness. The second role of this diagnostic covers the analysis of impurity line emission in visible wavelength range to provide more advanced insight in the plasma properties and behavior. Therefore, in addition to the robust continuum measurement, dedicated optical analysis instrumentation needs to be foreseen.

One of the concern for all of the optical diagnostics is stray light problem [1-4]. Since the wall will be comprised of metals (tungsten and beryllium), which have higher reflectance than that of carbon based materials, the reflection on the wall can be an important issue in ITER $[5,6]$. The reflection of light from walls will form phantom signal and disturb the real signal considerably for some diagnostics. Applicability of a couple of different methods to avert the stray light has been investigated. From detailed spectrum shape analysis, because the divertor stray light and emission from the scrape off layer had different spectrum shape, the stray light from the divertor was successfully estimated, and the actual signal was deduced in JET ITER like wall ar-

author'se-mail: kajita.shin@nagoya-u.jp rangement $[7,8]$. Transfer matrices prepared by ray tracing simulations were used to eliminate the stray light in synthetic ITER divertor emission [9, 10]. In addition to those inverse analysis approaches, an optical viewing dump can be a powerful tool $[6,11]$; it has been assessed that the stray light can be reduced roughly by an order of magnitude.

In the VSRS, it is planned to use a hole on the facing wall as an optical dump. Thus, it is expected that the VSRS will have a much lower stray light than other field of views (FOVs). In this study, the performance of the stray light reduction in the VSRS is investigated using a ray tracing simulation. One of the influential factors, the impact of which has yet to be known even for other diagnostics, is the variation of the reflection property of the first mirror due to the deposition and erosion [12]. By introducing full optics in addition to ITER vacuum vessel, the sensitivity of the reflection property on the first mirror is assessed using the ray tracing simulation.

\section{Model}

Figure 1 show a schematic overview of the VSRS conceptual design. The front-end optical components are installed in equatorial port 8 , and the collected light is transported to the diagnostic building using an optical fiber bundle. An inset in Fig. 1 shows the front-end optics (first and second mirrors). In front of the first mirror, an optical shutter will be equipped to protect optics. The VSRS sightline crosses the plasma tangentially and intersects the diagnostic neutral bean (DNB).

Figure 2 show a schematic of the developed model in the ray tracing simulation software LightTools (Synopsys, 


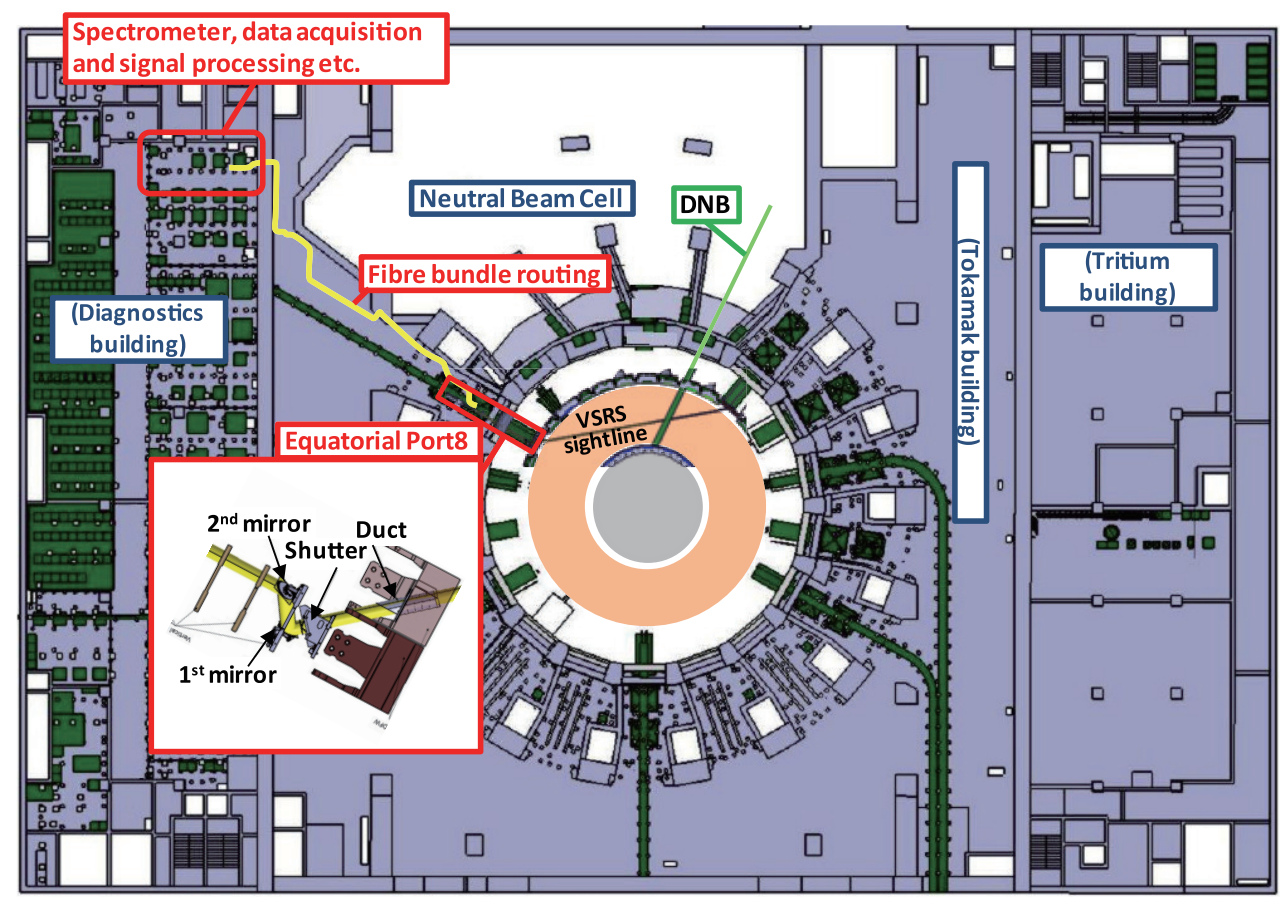

Fig. 1 Top view of a schematic overview of the VSRS conceptual design.

Inc.). A $120^{\circ}$ section of ITER vacuum vessel model with perfect mirrors on both of the boundaries was used. The model uses a part (1/3) of the whole vessel to save the computational memory used assuming that the emission from the plasma is toroidally uniform. Although a pinhole configuration has been frequently used to assess the stray light level in previous studies [6], the full set of collection optics was installed to the model to investigate the influence of the quality of the first mirror for the stray light reduction ratio. The collection optics were comprised of eight mirrors, and rays were launched from the fiber position toward the vacuum vessel with using the backward ray tracing method; in Fig. 2, some backward rays are presented. At the end of the FOV, a $60 \mathrm{~mm}$ radius retroreflector was embedded at the bottom of a $500 \mathrm{~mm}$ depth hole on a blanket module (stainless steel). The retroreflector is used for a dispersion interferometer and polarimeter (DIP) [13]. The VSRS will share the hole on the wall with DIP, which also has a line of sight from the equatorial port 8 . Concerning the radius of the hole that surrounds the retroreflector, we have preinvestigated the influence, and it was found that the stray light was not sensitive to the radius. Thus, in this model, we determine the radius to be $80 \mathrm{~mm}$, which was slightly larger than the mirrors for the retroreflector.

Figure 3 shows a schematic diagram of the last part of the collection optics with typical beams of rays to the fiber bundle. The final two mirrors (M7 and M8) are offaxis parabolic mirrors to collect beams to the fibers. In between the fiber and the final M8 mirror, we can install a retractable mirror, which connects between the calibration system and optics. The calibration of the FOV can be done using a lamp and a detector allocated behind the two off-

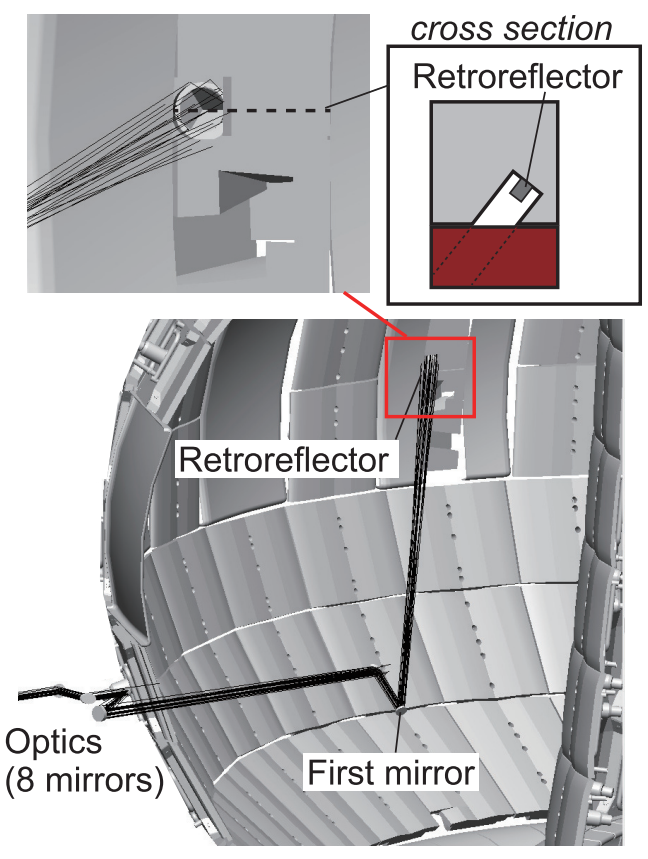

Fig. 2 Schematics of the model developed to assess the stray light for VSRS with some rays.

axis mirrors. In this study, we focus on the rays from the fiber bundle and do not use the calibration system.

The reflection property was defined by a mixture of specular and diffuse reflections except for seven mirrors of the optics (all but the first mirror), on which perfect mirror reflection was assumed. The specular reflection was defined by a Gaussian profile with a $1 / \mathrm{e}$ width, $w_{\mathrm{s}}$, and 


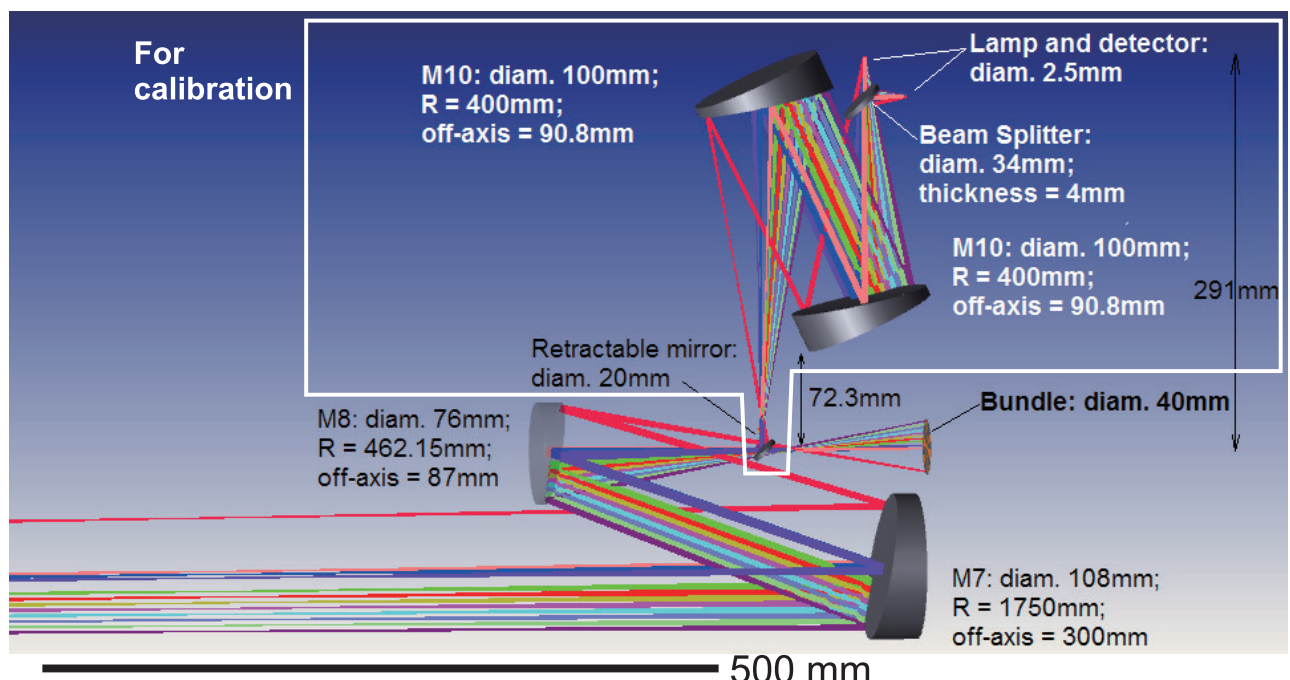

Fig. 3 A schematic diagram of the collection optics for VSRS after the front end optics.

the diffuse reflection was defined by the Lambertian distribution. The total reflectance, $R_{\mathrm{t}}$, which is the sum of the specular reflectance, $R_{\mathrm{s}}$, and the diffuse reflectance, $R_{\mathrm{d}}$, has a material dependence. In this study, we focused on the visible wavelength range, and it was assumed that $R_{\mathrm{t}}$ was $60 \%$ for blanket module (stainless steel), and $30 \%$ for first wall (beryllium) and divertor (tungsten). A lower $R_{\mathrm{t}}$ was chosen for beryllium and tungsten compared with that used in [6] (50\%). This was mainly because of uncertainty in actual $R_{\mathrm{t}}$, which could be changed by the surface morphology and the deposition/erosion effects. Since we will not discuss the absolute stray light level, $R_{\mathrm{t}}$ is not a sensitive parameter in this study. The width $w_{\mathrm{s}}$ was assumed to be $6^{\circ}$ for blanket module, first wall, and divertor, as following previous work [6]. A high density scenario used in [14] was chosen for the bremsstrahlung light source at the wavelength of $525 \mathrm{~nm}$. The density and temperature at the plasma center were $1.2 \times 10^{20} \mathrm{~m}^{-3}$ and $30 \mathrm{keV}$, respectively. The emissions profiles were represented using $~ 300$ toroidal shaped tubes in the same manner as in [14]. Only the emission from the core region (inside the last closed flux surface) was taken into account, considering the fact that the influence of the edge/SOL bremsstrahlung was one order of magnitude lower than that from the core [15].

\section{Results and Discussion}

\subsection{Influence of retroreflector mirrors}

Figures 4 (a-d) show images around the retroreflector with four different reflection properties for the retroreflector mirror with $R_{\mathrm{t}}=60 \%$ : (a) $R_{\mathrm{S}}=0 \%$ and $R_{\mathrm{d}}=60 \%$, (b) $R_{\mathrm{S}}=R_{\mathrm{d}}=30 \%\left(w_{\mathrm{s}}=1^{\circ}\right)$, (c) $R_{\mathrm{S}}=60 \%$ and $R_{\mathrm{d}}=60 \%\left(w_{\mathrm{s}}=1^{\circ}\right)$, and $(\mathrm{d}) R_{\mathrm{t}}=60 \%$ (mirror reflection). In Figs. 4 (a-d), the background plasma intensity is subtracted, and only the stray light component is presented. Three bright regions on the lower, left, and right sides of a
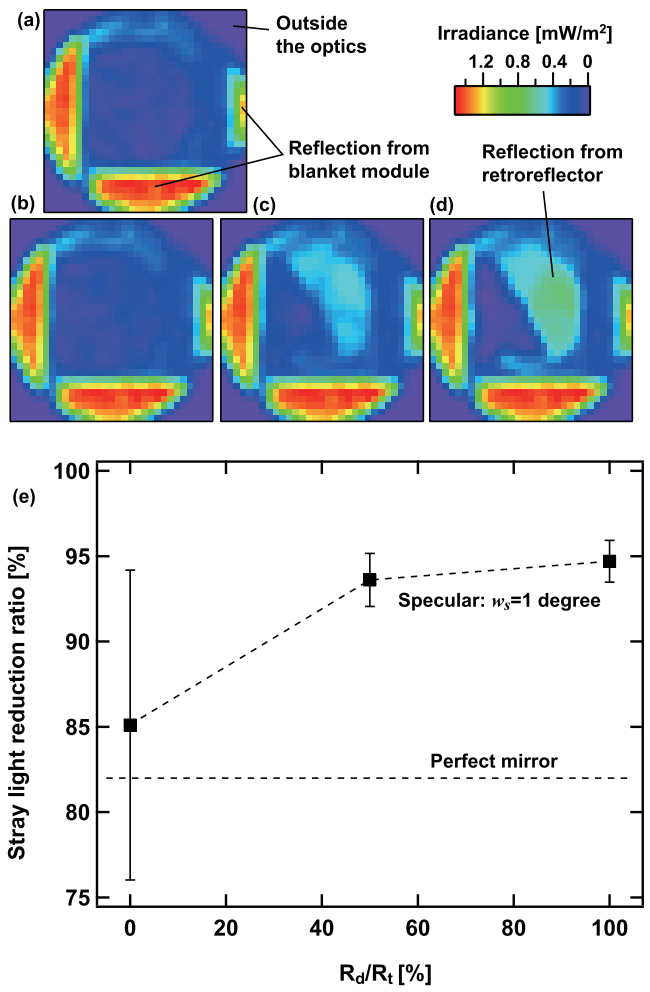

Fig. 4 (a-d) Images around the retroreflector for different reflection property of retroreflector material and (e) their summary: (a) $R_{\mathrm{S}}=0 \%, R_{\mathrm{d}}=60 \%$, (b) $R_{\mathrm{S}}=R_{\mathrm{d}}=30 \%$ $\left(w_{\mathrm{s}}=1^{\circ}\right),(\mathrm{c}) R_{\mathrm{s}}=60 \%, \mathrm{Rd}=0 \%\left(w_{\mathrm{s}}=1^{\circ}\right)$, and $(\mathrm{d})$ $R_{\mathrm{t}}=60 \%$ (mirror reflection).

dark circle, which corresponds to the retroreflector, are the reflection from the blanket module and wall. The intensity becomes zero outside the optics. The intensity in the central region is low in Figs. $4(\mathrm{a}, \mathrm{b})$, while it increases in Figs. 4 (c, d), suggesting that the stray light level increases with increasing of specular component. This is because the 
retroreflector can reflect back the emission from the plasma if the embedded three mirrors have mirror reflection.

The intensity around the central part, which corresponds to the retroreflector, is averaged, and the stray light reduction ratio (SLRR) is plotted as a function of $R_{\mathrm{d}} / R_{\mathrm{t}}$ in Fig. 4 (e). The SLRR was $82 \%$ when the retroreflector has perfect mirrors (Fig. 4 (d)). It increased with increasing the diffuse reflectance and was $95 \%$ when the retroreflector was totally diffuse. The results suggest that rough surface is more beneficial from the point of view of stray light.

Usually, since retroreflector will use mirror finish materials, the initial stray light ratio is likely $82-85 \%$. However, the reflectance could alter during discharges, as was demonstrated in the large helical device (LHD) [16]. In LHD, the reflectance of some retroreflector dropped to less than half in the visible range during the main and cleaning discharges in 160 days. Depositions and arc trails were observed on the retroreflector mirror surface. Similarly, the reflectance of retroreflector could deteriorate in ITER, even though they were embedded at the bottom of a deep hole. Thus, it is likely that the SLRR increases from $82 \%$ to $\sim 90 \%$ during discharges gradually. If the retroreflector is also used for interferometer, the reflectance can be roughly estimated from the reflected signal intensity when compensating for the wavelength dependence [16]. The actual SLRR in the visible wavelength may be assessed from the interferometer signal to obtain the stray light level.

\subsection{Variation of first mirror reflectance}

In Sec. 3.1, perfect mirror reflection was used for the first mirror, and the reflection property of the retroreflector was changed. During discharges and plasma cleaning process, changes in the surface roughness can lead to a change in the bidirectional reflectance distribution function (BRDF) of the first mirror. The BRDF can be expressed in general with following three components [17]: specular spike, specular lobe, and diffuse lobe. In this section, the influence of changes in the BRDF is investigated with changing the specular reflectance property and the diffuse reflection ratio.

Figures $5(\mathrm{a}-\mathrm{c})$ show the images with different reflectance properties of the first mirror: (a) $w_{\mathrm{s}}=0.01^{\circ}$, (b) $0.1^{\circ}$, and (c) $1^{\circ}$. It is noted that here we assume $R_{\mathrm{s}}=R_{\mathrm{t}}$ without any diffuse reflection, and the effect of the diffuse reflection is investigated later. Similar to Fig. 4, the stray light component is presented as subtracting the background plasma intensity. In this calculation, the reflection property of the retroreflector was assumed to be $R_{\mathrm{S}}=R_{\mathrm{d}}=30 \%$ with $w_{\mathrm{s}}$ of $1^{\circ}$. The image in Fig. 5 (a) was similar to the ones in Figs. $4(\mathrm{a}, \mathrm{b})$, showing clear images around the retroreflector. However, the dark region became smaller in Fig. $5(\mathrm{~b})\left(w_{\mathrm{s}}=0.1^{\circ}\right)$, and no dark region is identified in Fig. $5(\mathbf{c})\left(w_{\mathrm{s}}=1^{\circ}\right)$. The result suggested that only small divergence of the field of view distorts the image quality significantly. This is understandable, because the opening
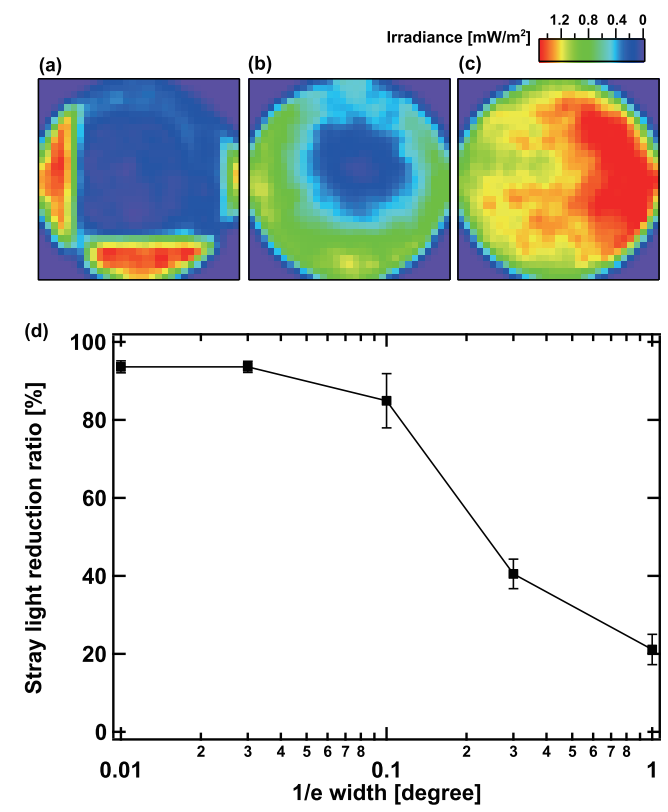

Fig. 5 (a-c) Images with different reflectance properties of the first mirror with $R_{\mathrm{s}}=R_{\mathrm{t}}$ : (a) $w_{\mathrm{s}}=0.01^{\circ}$, (b) $w_{\mathrm{s}}=0.1^{\circ}$, and (c) $w_{\mathrm{s}}=1^{\circ}$. (d) Stray light reduction ratio (SLRR) as a function of the $1 / \mathrm{e}$ width for the specular reflectance property.
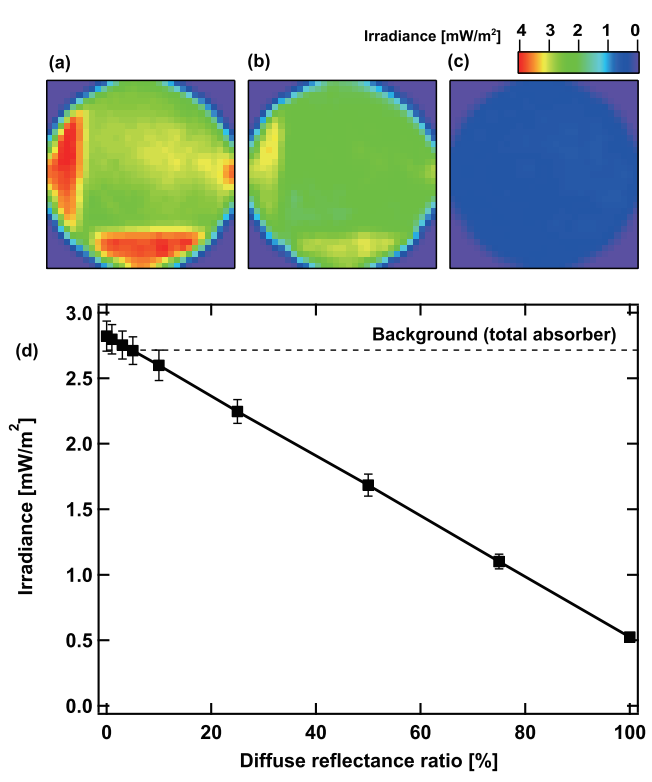

Fig. 6 (a-c) Images with different $R_{\mathrm{d}} / R_{\mathrm{t}}$ ratio of the first mirror at $w_{\mathrm{s}}=0.01^{\circ}$ : (a) $R_{\mathrm{d}} / R_{\mathrm{t}}=0 \%$, (b) $R_{\mathrm{d}} / R_{\mathrm{t}}=25 \%$, and (c) $R_{\mathrm{d}} / R_{\mathrm{t}}=100 \%$. (d) The irradiance as a function of $R_{\mathrm{d}} / R_{\mathrm{t}}$.

angle is $\sim 0.5^{\circ}$ when considering the distance from the first mirror to the retroreflector $(\sim 13 \mathrm{~m})$ and the radius of the retroreflector $(60 \mathrm{~mm})$. Figure $5(\mathrm{~d})$ shows the SLRR as a function of $w_{\mathrm{s}}$ of the first mirror. The SLRR is higher than $90 \%$ at $w_{\mathrm{s}}$ of $0.01^{\circ}$, and it decreases with increasing $w_{\mathrm{s}}$ when $w_{\mathrm{s}}$ is higher than $0.1^{\circ}$. When $w_{\mathrm{s}}$ is $1^{\circ}$, the SLRR decreases to $20 \%$.

Figures $6(a-c)$ shows the calculated images with dif- 
(a)

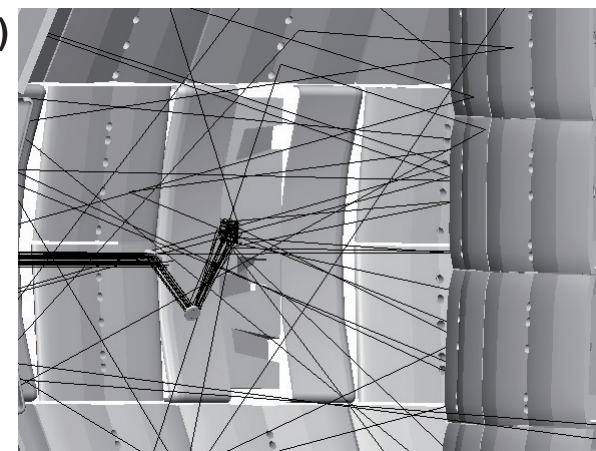

(b)

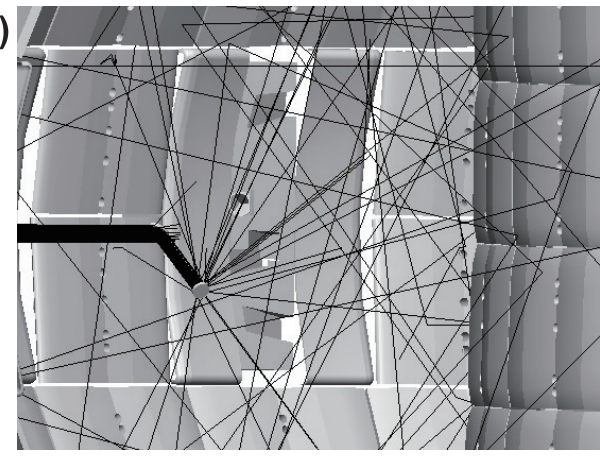

Fig. 7 Ray paths in typical two cases: (a) $R_{\mathrm{S}}=R_{\mathrm{t}}$ with $w_{\mathrm{s}}=$ $0.01^{\circ}\left(R_{\mathrm{d}}=0 \%\right)$ and (b) $R_{\mathrm{d}}=R_{\mathrm{t}}\left(R_{\mathrm{S}}=0 \%\right)$.

ferent diffuse reflectances for the first mirror: (a) $R_{\mathrm{d}} / R_{\mathrm{t}}=$ $0 \%$, (b) $R_{\mathrm{d}} / R_{\mathrm{t}}=25 \%$, and (c) $R_{\mathrm{d}} / R_{\mathrm{t}}=100 \%$. Here, different from the plots in Figs. 4 and 5, raw irradiance profiles are shown without any background subtraction. It is seen that the intensity gradually decreases with increasing $R_{\mathrm{d}}$. Figure 6(d) summarizes the irradiance on the receiver in the retroreflector region as a function of $R_{\mathrm{d}} / R_{\mathrm{t}}=0$. The intensity linearly decreases with $R_{\mathrm{d}} / R_{\mathrm{t}}$ and becomes much lower than the background level. The tendency is quite different from the specular case, where the intensity increases with $w_{\mathrm{s}}$. To understand the mechanism of this difference, we conducted ray path analysis. Figures $7(\mathrm{a}, \mathrm{b})$ show ray paths in two typical different cases: (a) $R_{\mathrm{S}}=R_{\mathrm{t}}$ with $w_{\mathrm{s}}=0.01^{\circ}\left(R_{\mathrm{d}}=0 \%\right)$ and (b) $R_{\mathrm{d}}=R_{\mathrm{t}}\left(R_{\mathrm{s}}=0 \%\right)$. When specular component is dominant (Fig. 7 (a)), all the rays from the first mirror arrive around the retroreflector. The lengths of rays before the first reflection are $\sim 13 \mathrm{~m}$. However, in diffuse reflection cases (Fig. 7 (b)), the rays are scattered from the first mirror and hardly reach around the retroreflector. Even considering reflection, the effective lengths of ray will be shorter than the original length. This is the reason for the reduction of the intensity with increasing diffuse component of the first mirror. It is noted, in reality, that the first mirror will be embedded in a port plug, and light can only be collected through a long tube ( $1.1 \mathrm{~m}$ in length and $60 \mathrm{~mm}$ in diameter), which is not implemented in this study for simplicity. Thus, the actual intensity would decrease by roughly $20 \%$ more than that in Fig. 6 (d) with increasing $R_{\mathrm{d}} / R_{\mathrm{t}}$ and to almost zero when $R_{\mathrm{d}} / R_{\mathrm{t}}=100 \%$.
First mirror will be subject to deposition and sputtering, which can deteriorate the mirror performance. Deposition decreases the specular reflectance significantly $[18,19]$, while the reduction by sputtering is likely less than $10 \%[20,21]$. In-situ cleaning is thought to be useful to remove the deposition. It was shown that the specular reflectance can be recovered after cleaning processes. However, it was also presented that diffuse reflectance gradually increased after repetitive cleaning processes [22], indicating that the surface roughness increased during the cleaning. It is likely that significant decrease in the total reflectance or specular reflectance is avoidable. However, it has yet to be revealed how the specular reflectance property changes.

Especially for a mirror finished surface, the specular spike is dominant and almost all the reflected power is within the half opening angle of $0.1^{\circ}[23,24]$. In Ref. [23], the specular peak measurements of steel with various roughnesses were conducted. The experiments well agreed with simulation results using the Modeled Integrated Scattering Tools (MIST). The intensity at $0.1^{\circ}$ decreased by five to eight orders of magnitude when the root mean square roughness was in the range of $6.5-130 \mathrm{~nm}$.

The present study suggested that it is necessary to carefully investigate the BRDF or width of the specular reflectance after many discharges or plasma cleaning processes; the threshold to change the image quality was around $w_{\mathrm{s}} \sim 0.1^{\circ}$. It is of interest to investigate how much the specular spike peak decreases after exposure to plasmas with high angular resolution, i.e. much less than $0.1^{\circ}$.

\section{Conclusions}

In this study, using the ray tracing simulation model developed with ITER vacuum vessel and the collection optics, the signal and stray light levels of the visible spectroscopy reference system (VSRS) in ITER were assessed.

The stray light level can be reduced by $80-85 \%$ in this field of view (FOV) compared with normal FOVs that see walls directly, because it has a $\sim 500 \mathrm{~mm}$ hole in the end of the FOV, which plays as a viewing dump. If the retroreflector has a diffuse reflectance, the stray light level can be reduced further. This is likely because the retroreflector mirror is less shiny during discharges due to deposition etc.

The impact of variation in the first mirror reflection was investigated. It was shown that a slight increase in the specular spike width could degrade the image quality significantly. Typically, when the $1 / \mathrm{e}$ width of the specular reflectance is greater than $0.1^{\circ}$, the hole does not work as a viewing dump anymore. It was shown that the first mirror would have diffuse component during the cleaning process. Different from the specular width widening, the diffuse components lead to the decrease in the signal or stray light level, because only the specular component can collect light from the plasma. Thus, although a slight increase of diffuse component would not have a strong im- 
pact, the width of the specular component could be influential for the measurement performance of the VSRS. It is likely that there are similar diagnostics that could be influenced by the first mirror specular performance such as laser Thomson scattering for the temperature and density measurements. It is of interests to investigate how the specular component, with high angular resolution much less than $0.1^{\circ}$, alters during the discharges.

\section{Acknowledgments}

This work was supported in part by a Fund for the Promotion of Joint International Research 17KK0132 from the Japan Society for the Promotion of Science (JSPS), and JSPS Bilateral Joint Research Project.

The views and opinions expressed herein do not necessarily reflect those of the ITER Organization.

[1] E.M. Hollmann, A.Y. Pigarov and R.P. Doerner, Rev. Sci. Instrum. 74, 3984 (2003).

[2] B. Schunke, G.T.A. Huysmans and P.R. Thomas, Rev. Sci. Instrum. 76, 083501 (2005).

[3] H. Meister, R. Dux, L.D. Horton, B. Kurzan, H. Zohm and P.J. McCarthy, Rev. Sci. Instrum. 74, 4625 (2003).

[4] S. Banerjee, P. Vasu, M. von Hellermann and R.J.E. Jaspers, Plasma Phys. Control. Fusion 52, 125006 (2010).

[5] M.-H. Aumeunier, M. Firdaouss, J.-M. Travère, T. Loarer, E. Gauthier, V. Martin, D. Chabaud, E. Humbert and J.-E. Contributors, Rev. Sci. Instrum. 83, 10D522 (2012).

[6] S. Kajita, E. Veshchev, S. Lisgo, R. Reichle, R. Barnsley, M. Walsh, A. Alekseev, A. Gorshkov, D. Vukolov, J. Stuber and S. Woodruff, Plasma Phys. Control. Fusion 55, 085020 (2013).

[7] A.B. Kukushkin et al., AIP Conf. Proc. 1612, 97 (2014).

[8] V. Neverov, A. Kukushkin, M. Stamp, A. Alekseev, S. Brezinsek, M. von Hellermann and J. Contributors, Nucl. Fusion 57, 016031 (2017).

[9] S. Kajita, E. Veshchev, R. Barnsley and M. Walsh, Contrib.
Plasma Phys. 56, 837 (2016).

[10] S. Kajita, E. Veshchev, M. De Bock, R. Barnsley, M. Von Hellermann and M. Walsh, Fusion Sci. Technol. 74, 37 (2018).

[11] E. Andreenko, A. Alekseev, A. Kukushkin, V. Neverov, S. Lisgo and A. Morozov, Fusion Eng. Des. 123, 825 (2017).

[12] A. Litnovsky et al., J. Nucl. Mater. 363-365, 1395 (2007).

[13] T. Akiyama, A. Sirinelli, C. Watts, P. Shigin, G. Vayakis and M. Walsh, Rev. Sci. Instrum. 87, 11E133 (2016).

[14] S. Kajita, M.D. Bock, M. von Hellermann, A. Kukushkin and R. Barnsley, Plasma Phys. Control. Fusion 57, 045009 (2015).

[15] S. Kajita, M.-H. Aumeunier, E. Yatsuka, A. Alekseev, E. Andreenko, A. Kukushkin, V. Neverov, M. Kocan, M. Bassan, E. Veshchev, M.D. Bock, R. Barnsley, A. Kukushkin, R. Reichle and M. Walsh, Nucl. Fusion 57, 116061 (2017).

[16] T. Akiyama, K. Kawahata, N. Ashikawa and M. Tokitani, Rev. Sci. Instrum. 78, 103501 (2007).

[17] S.K. Nayar, K. Ikeuchi and T. Kanade, IEEE Trans. Pattern Anal. Mach. Intell. 13, 611 (1991).

[18] A. Litnovsky et al., Nucl. Fusion 53, 073033 (2013).

[19] L. Moser, L. Marot, B. Eren, R. Steiner, D. Mathys, F. Leipold, R. Reichle and E. Meyer, Nucl. Fusion 55, 063020 (2015).

[20] T. Sugie, S. Kasai, M. Taniguchi, M. Nagatsu and T. Nishitani, J. Nucl. Mater. 329-333, 1481 (2004).

[21] B. Eren, L. Marot, M. Langer, R. Steiner, M. Wisse, D. Mathys and E. Meyer, Nucl. Fusion 51, 103025 (2011).

[22] L. Moser, L. Marot, R. Steiner, R. Reichle, F. Leipold, C. Vorpahl, F.L. Guern, U. Walach, S. Alberti, I. Furno, R. Yan, J. Peng, M.B. Yaala and E. Meyer, Physica Scripta 2017, 014047 (2017).

[23] V. Goossens, N. Gotzen, S.V. Gils, E. Stijns, G.V. Assche, R. Finsy and H. Terryn, Surf. Coat. Technol. 204, 551 (2009).

[24] K. Moeggenborg, F. Batllo, D. Mcmullen and S. Reggie, "Low-Scatter Optical Surfaces - Chemical-mechanical polishing shines mirrors", LaserFocusWorld 44, No. 6 (2008). 